\title{
Formación profesional, pymes e innovación en Navarra
}

\section{Vocational training, SMEs and innovation in \\ Navarre}

\author{
Mikel Olazaran ${ }^{1}$ \\ ENEKa AlBiZu ${ }^{2}$ \\ CRISTINA LAVÍA ${ }^{3}$ \\ BEATRIZ OTERO ${ }^{4}$ \\ Universidad del País Vasco (UPV/EHU) (España)
}

Recibido el 8 de junio de 2011, aceptado el 20 de febrero de 2012

$\mathrm{N}^{\mathrm{o}}$ de clasificación JEL: I20, J24, M53, 031

DOI: $10.5295 / \mathrm{cdg} .110290 \mathrm{mo}$

\section{Resumen:}

Los estudios recientes sobre sistemas de innovación no han prestado suficiente atención al papel que los centros de Formación Profesional y los trabajadores técnicos pueden jugar en los procesos de innovación. Este estudio parte de una revisión del estado de la cuestión en la literatura internacional y, en base a una encuesta a pymes industriales, completada con entrevistas cualitativas con responsables de centros de FP, pymes y actores institucionales, analiza la incidencia y el alcance de los distintos tipos de relación entre centros de FP y empresas en la Comunidad Foral de Navarra (una región con un alto nivel industrial y de Formación Profesional). Los resultados muestran que, si bien las funciones de preparación del capital humano e inserción en las empresas tienen un nivel notable, objetivos menos tradicionales de los centros de FP como la formación continua y el apoyo a la innovación tienen un alcance, a día de hoy, limitado. Por parte de las empresas, existe un reconocimiento incipiente del papel que los trabajadores técnicos pueden jugar en los procesos de innovación aunque, en general, el sistema de FP y el sistema de innovación se encuentran insuficientemente conectados.

\footnotetext{
- Investigación realizada dentro del proyecto "Pymes industriales en el sistema regional de innovación: Los casos de Euskadi, Navarra y Cataluña" (CSO2008-06520-C02-01) financiado por el Ministerio de Ciencia e Innovación. Quisiéramos mostrar nuestro agradecimiento también a las personas que han atendido amablemente nuestra demanda de colaboración para darnos su punto de vista sobre el funcionamiento de las relaciones entre las empresas y los centros de FP, así como a los revisores y la directora de Cuadernos de Gestión por su atención, amables comentarios y por las contribuciones efectuadas.

${ }^{1}$ Departamento de Sociología I, Escuela de Profesorado de San Sebastián UPV/EHU, Plaza Oñati, 3, 20018 Donostia-San Sebastián.mikel.olazaran@ehu.es

${ }^{2}$ Departamento de Economía Financiera II, E.U. de Relaciones Laborales UPV/EHU, B ${ }^{\circ}$ Sarriena s/n, 48940 Leioa (Bizkaia).eneka.albizu@ehu.es

${ }^{3}$ Departamento de Sociología I, Facultad de C.C.Sociales y de la Comunicación UPV/EHU, B ${ }^{\circ}$ Sarriena s/n 48940 Leioa (Bizkaia). cristina.lavia@ehu.es

${ }^{4}$ Departamento de Sociología I, Facultad de Ciencias Sociales y de la Comunicación, UPV/EHU, B ${ }^{\circ}$ Sarriena s/n, 48940 Leioa (Bizkaia). beatriz.otero@ehu.es
} 


\title{
Palabras clave:
}

Pyme, Formación Profesional (FP), innovación, Navarra.

\begin{abstract}
:
Recent studies within the innovation systems perspective have not paid enough attention to the role that Vocational Training (VT) centres and technical workers can play in innovation processes. In this paper we review the recent international literature on the issue and present an exploratory case study carried out in the Northern Spanish region of Navarre, which has a high level of both industrial activity and VT education. The study is based on quantitative and qualitative evidence from industrial SMEs and VT agents. The different types of relationships between VT centres and SMEs are analysed: formal education, in-company work training, hiring of graduates, continuous training, and innovation-related services. Results show that firms have a highly positive perception of VT centres' more 'traditional' missions (graduate education and employability), but more advanced and innovation-related missions have a limited reach right now.
\end{abstract}

\section{Keywords:}

SME, vocational training (VT), innovation, Navarre. 


\section{INTRODUCCIÓN}

Los principales informes internacionales sobre desarrollo económico en los países industrializados ponen de manifiesto la importancia de la formación de la población y, en particular, de la Formación Profesional. Según un reciente estudio del Cedefop (2010), la Formación Profesional puede convertirse en un motor de éxito y competitividad para las economías y sociedades europeas. El estudio confirma la centralidad de la Formación Profesional, entre otros, en el sostenenimiento del desarrollo económico, en el estímulo de la capacidad de innovación empresarial y en la mejora de la productividad. A pesar de ello, la OCDE (2009) advierte de que ésta es una de las principales debilidades de la economía española.

Navarra es una de las regiones punteras en el ámbito estatal en relación con los niveles de la educación de su población, así como en términos de desarrollo de la actividad innovadora. El objetivo de este artículo es estudiar, de un modo exploratorio, cómo son las relaciones entre los centros de Formación Profesional y las pequeñas y medianas empresas industriales (pymes) de la Comunidad Foral de Navarra y cómo contribuyen (o pueden contribuir) estas relaciones a la actividad innovadora de las empresas. La base empírica del estudio son las respuestas de 81 pymes industriales a un cuestionario telefónico y 34 entrevistas cualitativas a agentes del sistema de Formación Profesional.

En concreto, en este estudio se pretende dar respuesta a las siguientes preguntas de investigación:

- ¿Cómo se relacionan los centros de Formación Profesional (FP) y las empresas en Navarra y cuál es el alcance de los distintos tipos de relación?

- ¿Hasta qué punto realizan los centros de FP actividades como la formación continua y el apoyo a la innovación? ¿Qué potencialidades y dificultades existen al respecto?

- ¿En qué medida los trabajadores técnicos con cualificaciones intermedias efectúan aportaciones a los procesos de innovación de las pymes industriales?

Los principales motivos que nos han conducido a la realización de este trabajo son dos: conocer cuál es la aportación de la FP a la competitividad de las empresas navarras, y la inexistencia de estudios que analizan la relación entre centros de FP y empresas en la Comunidad Foral. Entendemos que este trabajo será de interés para: a) los responsables empresariales, que recibirán información sobre cómo obtener utilidad y hacer más efectivas las relaciones con los centros de FP; b) los responsables de los centros de FP, que recibirán retroalimentación sobre la actividad que desarrollan, permitiéndoles diseñar estrategias para la mejora de su servicio e interacción con las empresas; c) el Gobierno de Navarra, que podrá definir políticas más adecuadas para conseguir una mejor transferencia de conocimiento desde el sistema educativo al tejido empresarial; y d) los analistas y estudiosos de la relación entre educación e innovación, que encontrarán una investigación en la que se operacionaliza esta relación.

La estructura del documento es la siguiente. En el apartado segundo, exponemos el marco conceptual desde el cual hemos realizado esta investigación. En el tercer apartado se presenta brevemente el caso de la FP en Navarra. Posteriormente (apartado cuarto), se detalla la metodología empleada, así como las proposiciones que se pretenden contrastar. Los apartados quinto, sexto y séptimo presentan los resultados obtenidos respecto a los distintos tipos de relación entre centros de FP y empresas: formación reglada, formación continua e innovación. Finalmente, en el apartado octavo se señalan las principales conclusiones del estudio. 


\section{FORMACIÓN PROFESIONAL Y SISTEMA REGIONAL DE INNOVACIÓN}

La perspectiva del sistema nacional de innovación (SNI) sostiene que las instituciones sociales condicionan o canalizan la acción económica, en general, y los procesos de innovación, en particular (Lundvall, 1992; Nelson, 1993; Edquist, 1997). Dentro de esta perspectiva, la aplicación a nivel regional ("sistema regional de innovación", SRI) ha ganado una gran aceptación (Braczyk et al, 1996; Cooke y Morgan, 1998; Storper, 1997; Maskell y Malmberg, 1999; Cooke, Gómez y Etxeberria, 1997; Maskell, 2001; OECD, 2001). La perspectiva del SRI enlaza con un creciente interés por la importancia del entorno regional de la innovación, especialmente, para las pequeñas y medianas empresas (pymes).

Desde esta perspectiva, la innovación se concibe como un proceso de aprendizaje interactivo dentro de la empresa (entre áreas funcionales, niveles jerárquicos y personas en la organización) y entre ésta y otras organizaciones (clientes, proveedores, institutos de I+D, centros de formación, agencias públicas, etc.) dentro de un entorno institucional y cultural, donde las relaciones entre el sector público y el privado, entre el sistema educativo y el productivo, así como otros factores como el marco de relaciones laborales, el sistema financiero y la cultura industrial tienen una gran importancia. El proceso no es lineal, y está caracterizado por múltiples realimentaciones (por ejemplo, entre clientes usuarios de una tecnología y fabricantes de la misma) y por procesos continuos de interacción entre agentes heterogéneos.

Las características institucionales de una región y sus infraestructuras de conocimiento se consideran importantes condiciones básicas que pueden servir de estímulo para promover las actividades innovadoras (Isaksen y Asheim, 2003). Diferentes estudios destacan que la región es especialmente importante para el intercambio de conocimiento tácito y para la provisión de mano de obra cualificada, ambos mecanismos muy importantes en los procesos de innovación de las empresas pequeñas (Asheim y Coenen, 2005; Kauffmann y Tödtling, 2003; Grotz y Braun, 1997; Doloreux 2003; Gebauer et al., 2005). La proximidad geográfica adquiere una importancia esencial en el surgimiento de capacidades de innovación (Asheim y Gertler, 2005: 309-310). La confianza, la reciprocidad, los valores compartidos, las redes y las normas aceleran la transferencia de información y el desarrollo de nuevo conocimiento. En este sentido, las redes de colaboración interempresarial e interorganizacional son particularmente importantes en el caso de las pymes, que conforman la gran mayoría del tejido empresarial europeo.

La perspectiva del sistema de innovación ha señalado la importancia de las innovaciones incrementales ligadas a la actividad productiva habitual, a la adopción e implementación de tecnologías y a la interacción entre usuarios y productores de sistemas técnicos (Rosenberg, 1994; von Hippel, 1988). En esta línea, recientemente se ha señalado la existencia de dos "modos de innovación", el basado más directamente en la ciencia y la tecnología (modo STI, "Science, Technology and Innovation") y el basado en las actividades productivas habituales y el aprendizaje interactivo (modo DUI, "Doing, Using and Interacting") (Jensen et al, 2007, Lundvall y Lorenz, 2007). El modo de innovación STI, típico de sectores de alta tecnología, se caracteriza por la importancia del conocimiento codificado, del personal científico interno y de las relaciones con instituciones científico-tecnológicas externas. Por contra, en el modelo DUI, más relacionado con la innovación incremental, prima la interacción entre personas y entre departamentos, el intercambio de conocimiento 
tácito y la relación estrecha con usuarios o clientes ${ }^{1}$. No obstante, hay que señalar que se trata de dos "tipos ideales" o modelos que pueden coexistir en el mismo sector e incluso en la misma empresa.

Aunque se reconoce que el sistema de innovación está "enraizado" en el sistema de producción y en el "sistema de desarrollo de los recursos humanos" (Lundvall, 2002; Lundvall y Christensen, 2003), los estudios recientes realizados desde esta perspectiva se han centrado, en lo que al papel de las infraestructuras de conocimiento se refiere, en el estudio de centros tecnológicos y universidades, descuidando el papel que estructuras como los centros de formación profesional pueden jugar en relación con las pymes. El estudio del papel de los institutos técnicos de FP se retrotrae a los estudios sobre clusters y distritos industriales, siendo una referencia central a este respecto los trabajos de Stuart Rosenfeld sobre el papel sobre algunas regiones de Estados Unidos (Rosenfeld 1998a, 1998b). Este autor sugirió que los institutos de educación y formación profesional técnica están en mejor posición que las universidades para dar servicio a las pymes, y que en algunos sectores industriales existe un problema de falta de técnicos, y no tanto de ingenieros (Rosenfeld, 1998a., pp. 6 y 35). El papel que los institutos técnicos de FP pueden jugar en el desarrollo regional ha sido reconocido en trabajos más recientes (Curtain, 2004; Moodie, 2006; Veluzzi, 2010), aunque como señala este último autor (ibíd., p 205), dicho papel no ha sido suficientemente estudiado.

Como señaló Rosenfeld (1998a, pp. 9 y ss.) en su informe para la OCDE, los centros de FP realizan distintas funciones en relación con el sistema económico, como son: la educación reglada (formación de capital humano), la formación continua de trabajadores y los servicios técnicos a las empresas (pudiendo llegar al apoyo a las pymes en los procesos de innovación). En relación con esta última función, se menciona también el posible papel de animador de la cooperación interempresarial y la importancia de las relaciones con las asociaciones empresariales y agencias de desarrollo local. Esta taxonomía de misiones sigue estando aceptada, tanto en la literatura más reciente (véase, por ej., Gurthie y Dawe, 2004), y tal y como hemos podido comprobar en las entrevistas realizadas en Navarra, en la práctica de los propios agentes educativos.

Respecto a la educación reglada (función más tradicional de los institutos de FP), se señala la necesidad de que los centros educativos adapten su programación o curriculum a las necesidades de las empresas, de que dispongan de sistemas de prácticas y servicios de colocación de los egresados, y de que el personal de los centros tenga experiencia en la industria (Rosenfeld 1998a; Curtain 2004, pp. 45-46 y 53).

Por su parte, la formación continua sería, en orden de importancia, la primera misión "no tradicional" que realizan los institutos de FP. En los casos de las regiones norteamericanas avanzadas estudiadas, Rosenfeld (1998b, p. 18) señaló que la formación continua se había convertido en un elemento central de los programas educativos de los institutos técnicos, en base a financiación proveniente de agencias gubernamentales del ámbito económico o del empleo. La implantación de nuevas tecnologías en las empresas exige frecuentemente cambios en los procesos y en los sistemas de gestión que generan necesidades formativas que van más allá de la formación proporcionada por los proveedores de las

\footnotetext{
${ }^{1}$ Recientemente hemos mostrado la pertinencia del modelo DUI para comprender los procesos de innovación en pymes industriales españolas (Lavía, Otero, Olazaran y Albizu, 2011; Olazaran, Albizu y Otero, 2011).
} 
tecnologías. Por tanto, la inversión en nuevas tecnologías exigiría una inversión paralela en formación continua (Rosenfeld 1998a, pp. 6 y 16). En este sentido, estudios efectuados en el ámbito estatal (Rubio y Aragón, 2002) ponen de manifiesto que la formación continua y la innovación son factores que contribuyen a la mejora de la competitividad de las pymes.

La tercera misión (segunda misión "no tradicional") de los institutos de FP (los servicios técnicos a las empresas) está relacionada con la visión amplia de los procesos de innovación como procesos incrementales, de aprendizaje continuo, relacionados con las actividades productivas habituales y que afectan también a sectores de intensidad tecnológica media o baja, así como a empresas pequeñas y medianas. Este tipo de innovación se refiere a desarrollos, adaptaciones y recombinaciones de elementos técnicos existentes, así como a mejoras en los procesos, y no tanto a tareas de investigación básica o aplicada (Guthrie y Dawe 2004, p. 12; Moodie 2006, p. 6).

A este respecto, diversos estudios señalan la necesidad de coordinación entre los centros de FP y los centros tecnológicos. Tradicionalmente, los institutos técnicos han jugado un papel en la innovación y en el desarrollo local en momentos en los que las actividades de I+D formalizadas como tales apenas existían (Guthrie y Dawe 2004 señalan el caso australiano como ejemplo). El surgimiento de nuevos agentes de I+D, como son, los centros tecnológicos ("centros cooperativos" en Australia), que cubren nuevas necesidades, no debiera desligarse de los institutos de FP, que serían así "usuarios" de la investigación realizada por los centros tecnológicos, colaborando con los mismos en el diseño y desarrollo de nuevos sistemas y procesos (Whittingham, Ferrier y Trood, 2004; Guthrie y Dawe 2004). No obstante, estos autores reconocen que, en el caso australiano, no se han hecho esfuerzos suficientes para poner en relación a estas dos estructuras. En general, como señalan estos estudios, la participación de los centros de FP en misiones diferentes a las tradicionales se ve obstaculizada a menudo por diferentes barreras, como son: la falta de autonomía o flexibilidad de los institutos, la escasez de incentivos o de instrumentos públicos de fomento y la poca relación con otros agentes de $\mathrm{I}+\mathrm{D}$, tales como los centros tecnológicos.

La desconexión entre la Formación Profesional y la innovación se retrotrae, en última instancia, a factores culturales e institucionales. Como han indicado Bosch y Charest (2008, p. 430), mientras que en algunos países (el ejemplo típico sería Alemania) la posesión de una credencial educativa en FP es una señal de que el egresado/a en cuestión tiene capacidad para realizar autónomamente tareas complejas en un ámbito ocupacional amplio, en otros países dicho título es una señal de fracaso académico y de capacidad de realizar únicamente tareas específicas de baja cualificación ${ }^{2}$.

En el ámbito de las empresas y la organización del trabajo, se tiende a olvidar que los trabajadores técnicos con cualificaciones intermedias intervienen en el diseño, funcionamiento y mantenimiento de los productos y procesos, y por tanto contribuyen (o pueden contribuir) a la innovación en las empresas (Toner, 2009; Tether et al, 2005). Las empresas innovadoras necesitan una plantilla cualificada e implicada en la mejora continua de proce-

\footnotetext{
${ }^{2}$ La literatura institucionalista y de Economía Política muestra que los sistemas productivos, de relaciones laborales y de estátus nacionales, así como las políticas públicas, afectan de manera muy importante la concepción y organización de la Formación Profesional (ver por ej. Brinton, 2005; Brown, Green y Lauder, 2001; Estevez-Abe, Iversen y Soskice, 2001). Sin embargo, esta literatura apenas conecta con el estudio de los procesos de innovación en las empresas.
} 
sos y productos. Es más, como han mostrado Toner, Turpin y Woolley (2010), buena parte del personal de I+D registrado por las estadísticas habituales (46\% del personal de I+D empresarial en Australia) está formado por trabajadores técnicos cualificados procedentes de la Formación Profesional que no sólo realizan tareas de apoyo a los ingenieros o científicos, sino también tareas creativas y autónomas de resolución de problemas y de adaptación de las ideas propuestas por aquéllos.

Los obstáculos para una mayor participación de los trabajadores técnicos en los procesos de innovación pueden estar relacionados con la cultura y sistemas organizativos de las empresas. Recientemente se han realizado estudios que muestran un nivel de correlación importante entre las actividades de formación continua en las empresas europeas y la utilización de formas organizativas avanzadas o "aprendizaje discrecional", que sería vista como una dimensión de los procesos de innovación (Lundvall, Rasmussen y Lorenz, 2008; Lundvall, 2009). Estos trabajos apuntan la existencia de una divisoria Norte-Sur en Europa: las economías más avanzadas e innovadoras se caracterizarían por un mayor nivel de aprendizaje organizacional. Se indica que países como Portugal, España, Italia y Grecia, que han realizado importantes esfuerzos dirigidos a aumentar el número de graduados en ciencia e ingeniería, destacan por sus bajos niveles de actividades de formación continua y de aprendizaje organizativo (Lundvall, Rasmussen y Lorenz, 2008, p 689).

En suma, aunque en distintas instancias y discursos se reconoce que una estructura de cualificaciones técnicas intermedias más ampliamente distribuidas y de más nivel conceptual puede jugar un papel relevante en los procesos de innovación, tal y como indica Toner (2009, p. 61), se han hecho pocos trabajos en el campo de los estudios de la innovación, o de otras disciplinas, que lleguen a operacionalizar esta influencia. Algunos casos pilotos realizados mostrarían que los sistemas de organización participativos posibilitan que los trabajadores cualificados procedentes de la FP realizan aportaciones dirigidas a la mejora de productos y procesos, así como la satisfacción de las empresas con la formación (tanto reglada como continua) ofrecida por los centros de FP (Toner, Marceu, Hall y Considine 2004, pp. 98 y 101).

\section{LA FP EN NAVARRA}

La Comunidad Foral de Navarra cuenta con un nivel educativo de su población activa superior a la media española, que se refleja en mayores porcentajes de personas con formación secundaria postobligatoria y terciaria (véase la tabla 1).

Tabla 1

Población activa según nivel educativo: Navarra-España 2009 (\%)

\begin{tabular}{lcccccrrr}
\hline & $\begin{array}{c}\text { Anal- } \\
\text { fa- } \\
\text { betos }\end{array}$ & $\begin{array}{c}\text { Sin es- } \\
\text { tudios y } \\
\text { primarios }\end{array}$ & $\begin{array}{c}\text { Bachiller } \\
\text { elemental/ } \\
\text { EGB/ESO }\end{array}$ & $\begin{array}{c}\text { Bachiller superior/ } \\
\text { BUP Y COU/ } \\
\text { Bachillerato }\end{array}$ & $\begin{array}{c}\text { F.P. } \\
\text { Grado } \\
\text { medio }\end{array}$ & $\begin{array}{c}\text { F.P. Grado } \\
\text { superior }\end{array}$ & $\begin{array}{c}\text { Anteriores } \\
\text { al superior }\end{array}$ & Sup. \\
\hline C.F. Navarra & 0,1 & 9,2 & 26,3 & 14,2 & 11,4 & 14,3 & 11,3 & 13,2 \\
ESPAÑA & 0,5 & 14,8 & 28,6 & 15,2 & 8,8 & 9,9 & 9,4 & 12,9 \\
\hline
\end{tabular}

Fuente: Instituto Valenciano de Investigaciones Económicas (IVIE) 
Esta comunidad tiene, así mismo, una larga tradición en el campo de la formación profesional y sus relaciones con la empresa ${ }^{3}$. La Formación Profesional en Navarra cuenta con 28 centros ( 21 públicos, 6 concertados y uno no concertado). El número de alumnos/ as que cursa estos estudios es 6.104, de los cuales un 76\% lo hacen en la red pública (curso 2008-2009; datos del Ministerio de Educación). Las especialidades que concentran un mayor número de alumnos son: Administración (17,9\% alumnos en Grado Medio y 17,5\% en Grado Superior), Electricidad y Electrónica (13,0\% y 12,9\%), Mantenimiento y Servicios a la Producción $(9,3 \%$ y $11,8 \%)$, Sanidad (10,4\% y 8,4\%) y Fabricación Mecánica $(12,6 \%$ y $5,6 \%$ ). El profesorado de FP asciende a 922 personas, 726 en la red pública (43 de los cuales comparten docencia en FP, ESO y Bachillerato) y 196 en la red privada (37 con docencia compartida) (Consejo Escolar de Navarra, 2010).

En los últimos años se han llevado a cabo importantes reformas en el sistema español de Formación Profesional (Homs, 2008). Cabe mencionar a este respecto la implantación de los nuevos grados Medio y Superior a partir de la LOGSE, la institucionalización de los convenios con las empresas para la Formación en Centros de Trabajo (FCT) y la revisión de los planes de estudios en base a competencias y módulos profesionales. A partir de la leyes orgánicas 5/2002 y 2/2006 y sus desarrollos, siguen su curso en la actualidad los cambios dirigidos a la definición e implantación del Sistema de Cualificaciones y la integración de los distintos sistemas de formación (reglada, continua y ocupacional). Estos cambios responden a las directrices europeas (Lisboa 2000, Copenhague 2002 y Maastricht 2004), dirigidas a adaptar la formación y cualificación laboral a las demandas de los sectores productivos y a las necesidades de los trabajadores, dentro de una visión de "aprendizaje permanente" como elemento clave de innovación, competitividad y bienestar social en Europa.

En el ámbito de Navarra hay que mencionar el Plan de Formación Profesional 20012004, el Acuerdo de 4 de abril de 2005 del Gobierno de Navarra (por el que se establecen las directrices para el desarrollo del Sistema de Cualificaciones y Formación Profesional en Navarra) y el Decreto Foral 54/2008, que regula la ordenación y el desarrollo de la formación profesional en el sistema educativo de la Comunidad Foral. Entre los objetivos de este decreto están: el desarrollo del currículum educativo, la potenciación de las relaciones con la empresa (a través de la oferta de formación para trabajadores y la participación de profesionales del sector productivo como docentes) y el desarrollo de los centros integrados que serían, según la nueva normativa, referentes en sus especialidades tanto para formación reglada como para ocupacional y continua (Navarra fue pionera en su regulación en 2006), así como el impulso a la innovación tecnológica y didáctica y a la internacionalización de los centros de FP.

Dentro de este proceso de cambio del sistema de formación y cualificaciones, un aspecto clave es la relación entre los distintos sistemas de formación (reglada, ocupacional y continua), donde intervienen dos ámbitos de la Administración (Educación, por un lado, e Innovación, Empresa y Empleo, por otro) y donde desempeña un importante papel el Consejo Navarro de Formación Profesional, órgano consultivo y asesor en el que participan,

\footnotetext{
${ }^{3}$ Baste recordar que el centro decano de la FP en Navarra - el Colegio Salesianos de Pamplona- fue fundado en 1927 y que centros públicos de distintas comarcas navarras como el CIP ETI de Tudela, el CIP Virgen del Camino de Pamplona o el Politécnico de Tafalla superan ya los 40 o, en su caso, 50 años de trayectoria.
} 
junto con los departamentos mencionados, los agentes sociales ${ }^{4}$. En 2011 los presupuestos para formación ocupacional (preferentemente para desocupados) y continua (preferentemente para ocupados) en Navarra fueron, respectivamente 5 y 9 millones de euros (Presupuestos de Navarra 2011) ${ }^{5}$. En base a un convenio entre el departamento de Empleo y el departamento de Educación, los centros de FP han recibido 766.710 euros para su oferta de cursos de formación ocupacional y continua.

\section{METODOLOGÍA Y PROPOSICIONES A VALIDAR}

Este estudio de carácter exploratorio se basa en la combinación y triangulación de evidencia cualitativa y cuantitativa. Por un lado, se cuenta con evidencia procedente de 14 entrevistas con actores del sistema de $\mathrm{FP}^{6}$ y 20 entrevistas con responsables empresariales en pymes industriales de entre 50 y 175 empleados en sectores de media-alta tecnología ${ }^{7}$. Estas entrevistas cualitativas (semiestructuradas en profundidad) se realizaron presencialmente entre abril de 2010 y marzo de 2011, al objeto de conocer cuál es la visión de los actores del sistema regional sobre las relaciones que se vienen desarrollando entre los centros de FP y las empresas en Navarra.

Por otro lado, se ha encuestado a 81 pymes industriales navarras de entre 25 y 249 trabajadores (códigos CNAE93 entre 15 y 41). La encuesta se realizó a lo largo del mes de junio de 2010 de modo telefónico, entrevistando fundamentalmente a responsables de recursos humanos y formación (85\%) o, secundariamente, a gerentes o directores de las empresas. El contenido del cuestionario utilizado fue elaborado ad hoc tomando como referencias los objetivos de la investigación, la literatura analizada, los resultados indiciarios de la investigación cualitativa, y las limitaciones inherentes a la modalidad de encuesta seleccionada (telefónica).

Las encuestas realizadas constituyen la parte proporcional correspondiente a Navarra de una muestra de empresas de ámbito territorial mayor. Por lo tanto, aunque en cuanto a tamaño es una muestra estadística con limitaciones ${ }^{8}$, la selección de las empresas, de modo totalmente aleatorio y además proporcional a tamaños, contribuye a su representatividad y a la relevancia de los hallazgos empíricos. La siguiente tabla exhibe las características de la muestra.

\footnotetext{
${ }^{4}$ En Navarra participan en este organismo la Confederación de Empresarios de Navarra (CEN) y los sindicatos UGT y CCOO.

${ }^{5}$ En los presupuestos hay otras partidas relacionadas con la formación. Hay que destacar, por ejemplo, una partida de 5,560 millones de euros para escuelas-taller (programas mixtos de formación y empleo para jóvenes desempleados).

${ }^{6} 12$ directores de centros de Formación Profesional o responsables de relaciones con la empresa, un responsable del departamento de Educación del Gobierno de Navarra y el responsable de Formación de un sindicato. Estas entrevistas corresponden a la numeración FPx.

${ }^{7}$ Directores gerentes y directivos de oficinas técnicas, de departamento de I+D o personas con responsabilidades en innovación en las empresas entrevistadas. Estas entrevistas corresponden a la numeración Ex.

${ }^{8}$ El error muestral máximo es del 9,8\% para estimaciones a un nivel de confianza del 95, 5\%, en el supuesto de diversidad más desfavorable $(\mathrm{p}=\mathrm{q}=0,5)$
} 
Tabla 2

Principales características de la muestra $(\mathrm{N}=81)$

\begin{tabular}{ll}
\hline Sectores de actividad & \\
\hline Metalmecánica: $45,7 \%$ & Otros sectores industriales: $54,3 \%$ \\
\hline Tamaño & $150-249$ empleados: $7,4 \%$ \\
\hline 25-74 empleados: $75,3 \%$ & \\
$75-149$ empleados: $17,3 \%$ & \\
\hline Comarcas & Zona Navarra Media Oriental: $1,2 \%$ \\
\hline Zona Noroeste: $11,1 \%$ & Zona Ribera Alta: $16,0 \%$ \\
Zona Pirineo: $4,9 \%$ & Zona Tudela: $21,0 \%$ \\
Zona Pamplona: $39,5 \%$ & \\
Zona Tierra Estella: $6,2 \%$ & \\
\hline
\end{tabular}

Las principales proposiciones que pretendemos validar en este trabajo son las siguientes:

1) Los centros de FP de Navarra tienen un nivel muy importante de relación con el tejido empresarial (pymes industriales). El principal nexo de relación entre ambos agentes son las prácticas de formación en centros de trabajo (educación reglada) y los posteriores procesos de contratación de egresados. Estas relaciones son continuas y están basadas en la proximidad, la reciprocidad y la confianza mutua. De las mismas se derivan otros tipos de relación, así como una adaptación del curriculum por parte de los centros

2) La formación continua y los servicios técnicos, como formas de relación, están mucho menos extendidas. En particular, existen importantes barreras a un mayor desarrollo de la formación continua, tanto internas como externas al sistema de FP

3) En las empresas existe un cierto reconocimiento, no muy elevado, de las aportaciones de los trabajadores de FP a los procesos de innovación

\section{EDUCACIÓN REGLADA Y PRÁCTICAS DE FORMACIÓN EN CENTROS DE TRABAJO (FCT)}

Del total de empresas encuestadas, el $73 \%$ afirma que tiene o ha tenido alguna relación con centros de FP. La existencia de relaciones con centros de FP es significativamente más frecuente entre las empresas de mayor tamaño (a partir de 50 empleados el porcentaje asciende al 85,7\%) y entre las empresas de metalmecánica (86,5\%). Sin llegar a ser diferencias estadísticamente significativas, la detección de relaciones es también más frecuente entre las que se consideran innovadoras $(74,1 \%)$, y entre las que tienen un plan de formación $(76,8 \%)$. Creemos que este nivel de relación entre las empresas y los centros de FP es un hecho altamente significativo, que sugiere un grado importante de interacción entre las empresas y el sistema educativo de FP. 
Tabla 3

Existencia de relaciones con centros de $\mathrm{FP}(\%)(\mathrm{N}=81)$

\begin{tabular}{llcc} 
& & Sí & No \\
\hline \multirow{2}{*}{ Tamaño } & Hasta 50 & 63,0 & 37,0 \\
& Más de 50 & 85,7 & 14,3 \\
\hline \multirow{2}{*}{ Sector } & Metalmecánica & 86,5 & 13,5 \\
& Resto & 61,4 & 38,6 \\
\hline \multirow{2}{*}{ Empresa innovadora } & Sí & 74,1 & 25,9 \\
& No & 70,4 & 29,6 \\
\hline \multirow{2}{*}{ Empresa con plan de formación } & Sí & 76,8 & 23,2 \\
& No & 50,0 & 50,0 \\
\hline TOTAL & & $\mathbf{7 2 , 8}$ & $\mathbf{2 7 , 2}$ \\
\hline
\end{tabular}

Otro aspecto importante sobre las relaciones de las empresas con los centros de FP es que son geográficamente cercanas: el $73 \%$ de las empresas se relacionan principalmente con centros de FP radicados en la misma comarca y el 19\% con un centro de otra comarca pero radicado en Navarra (por tanto, el 92\% de las relaciones se refieren a centros ubicados dentro de la propia Comunidad Foral, un indicador de su contribución al desarrollo regional).

De acuerdo con la evidencia cualitativa, recogida tanto en las empresas como en los centros de FP, se manifiesta una buena valoración de la capacitación del alumnado de FP, muy notablemente del nuevo Grado Superior surgido de la LOGSE, que compite por su alto nivel de adaptación a las necesidades de las pymes y por sus inferiores costes con los ingenieros ${ }^{9}$.

"En las empresas nos dicen que para ellos, desde la perspectiva del mando intermedio y de ser gente que tenga un feedback con la empresa, tienen más confianza en este tipo de técnicos [de grado superior], que en los que vienen de la universidad, que tienen, lógicamente, unos perfiles muchísimo más amplios en cuanto a lo que sería la especialización... Llevamos ya diez años con antiguos alumnos, y muchos de ellos están ocupando cargos relevantes en las empresas". (FP3, pp 16-17)

"En una Oficina Técnica prefieren muchas veces un delineante industrial antes que un ingeniero" (FP5, p 17)

"Mientras que un técnico de grado medio se ocupa de la conducción de una línea, el de grado superior es gestión en sí, de toda la planta. Serían los responsables de la calidad, los responsables de la logística, qué hacemos, a quién compramos, controlar la calidad, cómo te vienen los productos, calidad con la que salen. Muchos de nuestros alumnos se colocan en calidad. Muchos." (FP7, pp 20-21)

"La tendencia es a coger gente de grado superior [frente al grado medio]. En muchas ocasiones, huyen un poco del ingeniero, aunque lo utilizan a veces para hacer ese desempeño de un profesional, digamos, cualificado y demás. Las empresa buenas, competitivas de la zona están pensando en coger sólo a técnicos de grado superior.

\footnotetext{
${ }^{9} \mathrm{~A}$ este respecto existe un riesgo de que crezcan las distancias entre los grados superior y medio, bajando los estándares de calidad y alumnado de éste.
} 
Y también quieren, lógicamente, pagarles cuanto menos mejor. Tienen que ajustar costes, tienen que ser competitivos, y eso no sé si es justo o no, pero es una necesidad que tienen, el ser competitivos. Y si no lo son, al final se quedan sin trabajo. Y conjugar todo eso no es tarea fácil." (FP13, p 15)

"Los directivos no son ingenieros, ¿eh?. El Director General era un FP instalador de neumática y X lo mismo. Buenos técnicos. Ésos han nacido de buenos técnicos de FP. Increíblemente. Y se nota en la naturaleza de la empresa, y la gente” (E9, p.18)

El desarrollo de la función de formación de capital humano adaptado a las necesidades de la industria regional está, en buena medida, condicionado por la interacción centro-empresa. En esta interacción, con impacto en el rendimiento académico (curriculum) y diferido (empleo) del alumnado de FP, hay dos elementos que hemos considerado en este estudio: las prácticas de Formación en Centro de Trabajo (FCT) y la gestión de las Bolsas de Trabajo.

Tabla 4

Contribución de la relación FP-empresa a la educación reglada ( $\mathbf{N = 5 9 )}$

\begin{tabular}{lcc}
\cline { 2 - 3 } & Relación (\%) & $\begin{array}{c}\text { Satisfacción } \\
(0-10) \text { (Media) }\end{array}$ \\
\hline Recibimos alumnado en prácticas & 89,8 & 7,54 \\
Contratamos trabajadores/as a través de sus Bolsas de trabajo & 55,9 & 7,50 \\
\hline
\end{tabular}

La forma más generalizada y básica de relación de las pymes con los centros de FP es la de recibir alumnado en prácticas: de las empresas que declaran mantener relaciones con los centros, el $90 \%$ recibe alumnado en prácticas de FCT. Se trata, por tanto, de un tipo de relación ampliamente extendida y aceptada. Además, la recepción de alumnado en prácticas se valora con un elevado grado de satisfacción, y de manera homogénea, por parte de las empresas: 7,54 puntos en una escala 0-10.

Frecuentemente, las prácticas de FCT, que se realizan en el último trimestre del segundo año del ciclo de formación reglada (de Grado Medio o Superior), suelen estar relacionadas con la posterior contratación de los estudiantes. La contratación de trabajadores a través de las Bolsas de Trabajo de los centros es la segunda forma de relación más frecuente entre centros de FP y pymes industriales navarras. La mayoría de las empresas relacionadas con centros de FP (56\%) manifiesta que contrata trabajadores recurriendo directamente a los propios centros, lo cual supone una incidencia del $41 \%$ sobre el total de pymes encuestadas. Con una valoración media de 7,5 (escala 0-10), se deduce que también el grado de satisfacción de las empresas que recurren a este servicio es alto.

Cabe destacar que, entre todas las combinaciones de tipos de relación con centros de FP, la más frecuente es la que abarca los dos principales formatos analizados, es decir, recibir alumnado en prácticas y contratar a través de las bolsas de los centros: el 50,8\% de las 59 empresas que se relacionan con algún centro de FP lo hace de ambas formas y prácticamente el $95 \%$ tiene contacto con los profesionales preparados por los centros de una u otra forma. 
Por medio de las entrevistas cualitativas realizadas hemos podido comprobar que el sistema de prácticas FCT es el nexo principal de relación entre el sistema educativo de FP y las empresas. De la relación entre pymes industriales y centros de FP en torno a las prácticas se derivan resultados como la contratación de estudiantes egresados, adaptaciones en el curriculum de la formación reglada, detección de necesidades de formación continua y de necesidades tecnológicas.

"El profesor previamente ha ido a acordar un programa formativo con la empresa. Han llegado a un acuerdo en las tareas que van a realizar durante ese tiempo los alumnos que van a ir allí, hace un seguimiento quincenal, in situ, para ver cómo se va desarrollando todo este proceso, tiene contacto con los encargados de la empresa, etc, etc. Todo eso es una fuente de información directísima y tremenda" (FP11 p 3)

"Aquí, en el centro X, participan como tutores de prácticas FCT todos los profesores que imparten clase en el curso. Hay unos que llevan a dos alumnos, otros a tres, otros... De tal forma que todo el equipo está en contacto con la empresa... Con lo cual, quiere decir que todos se enriquecen de ir hacia el exterior y visualizar qué es lo nuclear, qué es lo fundamental, para luego aquí no quedarte con lo accesorio. Y luego, pues ese intercambio, esa puesta en común..." (FP3, pp 5-6)

"Conozco mucho a su director y a la gente que trabaja allí, y vienen a aquí a visitarnos, y traen a los chavales, y tal... Y siempre me intentan vender que ellos también desarrollan [tecnología], y que ellos también hacen, y que les gustaría [participar en proyectos]" (E10, p.9)

De las relaciones regulares entre los tutores de los centros y los instructores de las empresas (no hay que olvidar, además, que éstos son en ocasiones antiguos alumnos de los centros) surgen relaciones de cercanía y confianza de las que se deriva la contratación de egresados procedentes tanto de la FCT como de la Bolsa de Trabajo en general. El nivel de colocación era muy alto y el paro prácticamente inexistente hasta el estallido de la actual crisis.

"Enpresa askok deitzen digute guri lan eskaintza bat baldin badute. Guk ere lan poltsa badugu, zorionez nahiko hutsa dagoena, baina badugu, eta guregana bideratzen dute ze, beno, konfidantza badute azken finean, ze Nafarroako enplegu zerbitzu INEM delako horretatik ba edonor bidali dezakete. Orduan, badakite guk halako harremana, halako institutu txikia izanik, ba beno, badakite guk azken finean ezin diegula haiei gol askorik sartu. Guk ezin duguna da enpresa bati ba ikasle txar bat bidali, zer gero hortik buelta [dator]. Horregatik ba askotan deitzen digute guri" (FP2, p 2)

"O sea, intentas abordar un poco esas tres cosas. Primero, el tema de la formación, buscar la empresa ideal para hacer la FCT. Segundo, partir de la petición de trabajo de la empresa, y satisfacer a la empresa. Tercero, con un chaval que quiera trabajar y que se ajuste un poco. Esas tres cosicas, intentarlas encajar" (FP4,p 4)

"Nos hemos estado moviendo alrededor de 400 peticiones de trabajo al año. Al año salen 120 y 150 chavales y chavalas que terminan los estudios. Con lo cual, teniendo unas 400 peticiones, te puedes imaginar que, prácticamente, el paro era inexistente hasta octubre de 2008. A partir de esa fecha [debido a la crisis económica] el número de peticiones ha descendido radicalmente" (FP4, p 3)

"El 90\% de la gente que está trabajando aquí en la empresa ha pasado por ese centro. Han estudiado allí. Y ahora tenemos una relación muy estrecha." (E10, p.6) 
Una consecuencia tremendamente importante de las relaciones entre centros de FP y empresas que se establecen a través de la FCT es la realización, por parte de los centros, de adaptaciones en el curriculum de la formación reglada. Esta posibilidad, contemplada en la normativa legal (Decreto Foral 54/2008), supone un importante mecanismo de ajuste a las demandas de las empresas y se ha visto reflejada con profusión en las entrevistas realizadas.

"Y estos módulos formativos atienden a estas actividades, a este perfil, que nosotros tenemos aquí en el centro. Claro, a partir de ahí, sí que es cierto que el perfil después hay que circunscribirlo a la tipología de industria que haya en cada comarca, o en cada zona... [Por otro lado,] en cuanto a lo que sería inversiones en material para las prácticas y demás, pues sí que se tiene muy en cuenta también qué es lo que se está haciendo alrededor en las empresas" (FP3, pp 3-4)

"Hicimos un estudio, me encargué yo mismo de ver, antes de poner el ciclo formativo, qué necesidades tenía la zona. E, invariablemente, en todas las empresas del sector, se decía: necesitamos aquí una persona que no sólo sepa conducir las líneas de X, sino que sepa mantenerlas. Porque en cualquier momento hay un fallo, y no se puede esperar a que el taller auxiliar venga, porque se ha perdido mucho tiempo, porque igual es una tontería y un trabajador puede arreglar la máquina, ¿no? Y nosotros lo incorporamos y lo hicimos. Entonces, es un ciclo especial. No hay en toda España el ciclo que hay aquí. Es X más mantenimiento. Aumenta un año más los estudios” (FP7, p 4) "Tenemos una serie de equipos docentes, tenemos una serie de antenas diseminadas por todo el entorno productivo de $\mathrm{X}$ y su comarca, y eso nos permite captar cuáles son las tendencias, qué necesidades está teniendo ahora mismo la empresa, y ver y contrastar si realmente aquello que estamos intentando desarrollar en el propio alumnado es útil a la empresa, encaja, cumple con las expectativas" (FP13, p10)

Las relaciones entre centros de FP y empresas que se establecen a través de las prácticas de FCT son la base de formas más "avanzadas" de relación, entre las que destaca, en el caso de Navarra, la formación continua.

"A través de esos contactos [FCT] se derivan también, muchas veces, peticiones de las empresas, que te dicen: oye, ¿vosotros dais formación [continua]?”. (FP4, p 6)

"Las necesidades de formación continua de las empresas se detectan a partir de las prácticas y, sobre todo, de las estancias [de profesores en las empresas]. Y son las dos fuentes, básicamente" (FP10, p 15)

"Cuando hacemos las prácticas, pasamos sistemáticamente a las empresas una encuesta en la que detectamos, entre otras cosas, qué necesidades formativas serían interesantes para sus propios trabajadores y, en la medida de lo posible, canalizamos esa propuesta de actividades formativas a la Unidad Técnica de Formación Profesional [del departamento de Educación], para que nos facilite el que podamos impartir algún curso a las empresas a la carta, y/o haciendo un compendio de las distintas organizaciones" (FP13, p 1)

Una forma importante de relación entre los centros de FP y las empresas son las estancias de profesores en las mismas. Este instrumento, apoyado por el gobierno regional, es bien valorado como forma de profundizar en el conocimiento de las tecnologías y las necesidades de las empresas, pero se reconoce mayoritariamente que su extensión es, a día de hoy, limitada. Como en el caso de otras actividades consideradas "extras" del profesorado (formación continua, innovación), existen inercias en contra de su desarrollo, que depende más bien de iniciativas personales. 
"En esto, lo que te he dicho, estamos dos profesores de estancias en empresa. De los 20 , es un $10 \%$. El resto no está. Y bueno, aunque vamos pasando a lo largo de los años, pues en los últimos cuatro años, igual hemos pasado, pues dos, cuatro, seis o siete. Pero claro, el resto aún no ha pasado" (FP10, p 24)

"Ha estado puntualmente algún profesor, no es significativo" (FP12, p 12)

“¿Innovación? Casi prefiero que nos dejen ir a la empresa a trabajar, para ver qué está aplicando la empresa" (FP12, p 15)

Otras formas de interacción y mutua adaptación entre los centros de FP y las empresas es la participación de éstas en diferentes órganos de los propios centros de FP, como pueden ser, los patronatos de los centros (si son fundaciones) o asociaciones de antiguos alumnos, así como la creación de empresas, que históricamente ha tenido un papel importante en el desarrollo regional.

"En la Asociación de Antiguos Alumnos hay muchos antiguos alumnos que han creado pymes y continúan teniendo una relación con el centro. Valoran el centro, valoran la educación en valores, el proyecto educativo." (FP5, p 17)

"En veinte años (los antiguos alumnos) han montado un montón de empresas en la zona. Pequeñas, pero que nos dan salida para los alumnos nuestros [para la FCT] de maravilla." (FP6, p 13)

"Los chavales quieren salir, quieren buscar un puesto, un puesto que se gane bien, y dejarse de complicaciones. Y formar una empresa es una cosa seria. La antigua Formación Profesional, hace veinticinco años, sacabas gente a las empresas, y de ahí salían otras empresas, otras, otras...” (FP7, p 23)

\section{FORMACIÓN CONTINUA}

Los centros de FP aparecen como proveedores de considerable importancia en servicios de formación continua para las empresas con las que se relacionan: el 30,5\% de las pymes industriales navarras que tienen relaciones con los centros de FP envía a sus trabajadores a recibir formación continua reglada en los centros, lo que supone un 22,2\% del total de empresas encuestadas. Además, el $24 \%$ de las empresas ha desarrollado formación continua bajo demanda con centros de FP. Si bien la combinación de ambos formatos no es muy frecuente (19\%), sobre el total (59), un 36\% de las empresas que tienen relaciones con centros de FP ha recurrido a sus servicios de formación para trabajadores de una u otra forma, lo que constituye la tercera forma de relación más frecuente entre empresas y centros.

Tabla 5

\section{Contribución de la FP a la Formación Continua $(\mathrm{N}=59)$}

\begin{tabular}{lcc}
\cline { 2 - 3 } & Relación (\%) & $\begin{array}{c}\text { Satisfacción } \\
(0-10) \text { (Media) }\end{array}$ \\
\hline $\begin{array}{l}\text { Nuestros trabajadores/as acuden al centro para recibir cursos } \\
\text { de Formación Continua (Catálogo Modular) }\end{array}$ & 30,5 & 7,56 \\
\begin{tabular}{l} 
Hemos realizado con el centro Formación Continua “a la carta” \\
\hline
\end{tabular} & 23,7 & 8,07 \\
\hline
\end{tabular}


Los cursos de formación que imparten los centros FP para los trabajadores de pymes son muy bien valorados por las empresas: cuando se ha detectado este tipo de relación las valoraciones resultantes nunca son negativas, de manera que como imagen global tenemos un notable. La media de valoración de esta relación es de 7,6, subiendo hasta el 8 (sobre 10) en el caso de la formación a medida, una forma de relación y servicio menos frecuente pero más intensa: prácticamente todas las empresas (el 93\%) que realizan este tipo de formación continua tiene ya otros dos o más tipos de relaciones diferentes con los centros, incluyendo recibir prácticas, contratar, etc.

Las entrevistas cualitativas realizadas nos permiten profundizar en la problemática de la formación continua para los centros de formación profesional. La conclusión principal que se obtiene de esta evidencia empírica es que existen importantes obstáculos, tanto internos al sistema de FP como externos al mismo, para alcanzar la integración entre la formación reglada y la formación para el empleo, un objetivo clave de las reformas y normativas recientes (en el caso de Navarra, el Decreto Foral 54/2008).

En el momento de la realización del trabajo de campo en Navarra (entre abril y noviembre de 2010), existía una percepción unánime entre los agentes de FP entrevistados sobre la insuficiencia de los fondos asignados por el Servicio Navarro de Empleo (SNE) para cursos de formación continua en los centros de FP. De hecho, estos fondos fueron eliminados en 2009, debido a las desavenencias entre el departamento de Educación y los agentes sociales del Consejo de Formación Profesional. Los agentes de FP entrevistados manifiestan que, en comparación con los centros formativos creados al albur de las organizaciones sindicales, su oferta formativa y sus instalaciones se adaptan mejor a las necesidades de las empresas, y que podrían impartir mucha más formación continua de la que imparten en la actualidad (los centros entrevistados imparten por término medio entre 5 y 10 cursos anuales subvencionados por el SNE).

“A diferencia de la C.A. del País Vasco, aquí en Navarra, tienen mucho protagonismo los sindicatos y las organizaciones empresariales. Entonces, de hecho, prácticamente la totalidad de la formación continua va por ese conducto. Y una pequeñísima parte -mínima diría yo- es la que se deriva a los centros de formación profesional" (FP3, p 6) "No nos cabe la menor duda que realmente los que tendríamos la clientela seríamos nosotros, por la especificidad de cada una de las familias profesionales. Merecería la pena que los medios que tenemos estuviesen utilizándose desde las ocho de la mañana hasta las diez de la noche, y cuanto antes se amortizasen y se renovasen, pues todo el mundo saldría beneficiado. Y el hecho de estar en esos mismos centros, pululando los trabajadores y nosotros, pues habría esa ósmosis entre un sistema y el otro, que favorecería a todo el mundo" (FP3, pp 7-8)

"El problema es quién es el que administra eso [el sistema de formación continua]. Ése es el problema. Quién es el que administra y a quién se le adjudica" (FP4, p 10) "Formación continua se hacía antes más. ¿Por qué? Porque siempre había empresas que nos solicitaban, o nosotros ofertábamos cursos. Pero ahora los sindicatos son los que absorben. Todo el tema se ha ido para los sindicatos. Los sindicatos han asumido esas funciones, que no son propias de ellos. Y a nosotros nos han quitado el dinero" (FP6, p 6)

"No podíamos impartir un curso de soldadura, porque no nos lo concedían, y luego venía el sindicato $X$ con el curso de soldadura a ver si lo podían impartir en nuestras aulas" (FP8, pp 29-30) 
"En los años ochenta se llamaba Educación para Adultos (EPA), y desde este centro se organizaba formación continua para 1.800 trabajadores y trabajadoras. Educación para Adultos estaba insertado en el organigrama del centro, y se organizaban por todas las localidades, cursos... En la comarca antes teníamos la exclusividad, no había otro centro de formación profesional. La realidad de hoy es que, de diez años a esta parte, han emergido otros centros paralelos en materia de formación profesional, y tenemos una concurrencia competitiva yo entiendo que desleal... Nos está ocurriendo que algún curso de formación para el empleo que estamos ofertando nosotros, también lo están ofertando esas tres o cuatro entidades formativas de los sindicatos que existen en la zona. Y unos u otros nos vemos sin alumnos, porque no es tanto el potencial de gente dispuesta a formarse. Entonces, todo eso se tiene que coordinar. (FP13, pp 2-5)

Además de las dificultades relacionadas con los procesos de asignación de los fondos de formación continua, existen otros problemas de carácter más interno al sistema educativo de FP, como son la falta de autonomía de los centros, la falta de incentivos, los límites legales a la dedicación del profesorado funcionario a estas actividades y la imposibilidad de planificar e integrar la formación continua en los horarios y en la carga lectiva del profesorado titular. Incluso en el centro nacional integrado entrevistado, con programas de formación para el empleo de ámbito estatal, se constata una rigidez en la separación entre formación reglada y formación continua.

"En estos momentos, lo que sería la estructura de centros integrados, que fundamentalmente daría un poco respuesta a todo eso, con esas dimensiones, pues está muy poco desarrollada. Quiero decir, en el IMH [Instituto de Máquina-Herramienta, Elgoibar] hay gente que su jornada laboral la dedica a eso. Aquí eso es impensable. La formación continua siempre es como una cosa extra" (FP3, pp 10-11)

"Los cursos de 2010, solicitados en abril del año pasado, nos los han comunicado la semana pasada [abril de 2010]. Como comprenderán, nos queda mayo, junio -para este trimestre-, y luego, septiembre, octubre, noviembre y diciembre para el que viene. Para intentar encajar los once cursos que nos han concedido. Entonces, es muy difícil, y además la única posibilidad de ajustarlo ahí, en el centro, es hacer horas extraordinarias los profesores. Es decir, es el profe el que tiene que coger y decir: 'yo doy ese curso'. Entonces, un trabajo extraordinario, cosa que no es lo ideal. Lo ideal sería saber desde el principio la planificación, decir: 'tenéis estos cursos', y te puedes planificar desde los propios departamentos... puedes tener la planificación de los cursos reglados, y los cursos de formación continua u ocupacional.” (FP4, pp 7-8) "El centro integrado se ve de alguna manera con las manos atadas a la hora de hacer una oferta autónoma. ¿Por qué? Porque las partidas económicas dependen de Trabajo o, en todo caso, del departamento de Educación, que hace una redistribución entre todos los centros de su red pública... No tenemos capacidad para hacer una oferta directa en formación ocupacional y continua" (FP10, pp 3 y 6)

"Nosotros, al final, siempre, lo que se ha intentado luchar es por que te den medio profesor más, o te den algo, para que des esas horas. O cogerte y decir: 'bueno, pues vas a tener dos horas menos todos los días, y en la temporada que había que dar el curso, pues das el curso, y ya está'." (FP8, p 17)

A juicio del agente social entrevistado, un problema clave que obstaculiza una mayor financiación para formación continua en los centros de FP, principalmente, los centros inte- 
grados, es la falta de autonomía de los centros y su subordinación excesiva al departamento de Educación. Este problema se refleja en la falta de operatividad de los consejos sociales de los centros integrados, donde están representados los agentes empresariales y sindicales. Estos agentes exigen una mayor participación como condición para vehicular más recursos económicos hacia la formación continua en centros de FP.

En cuanto a la formación de demanda desde las empresas, según el sistema de deducción de las cuotas de formación por parte éstas, gestionado desde la Fundación Tripartita (bonificaciones), está menos extendida debido también, en parte, a las rigideces internas de funcionamiento de los centros públicos de FP. Algunas asociaciones empresariales comarcales están tratando de fomentar este tipo de formación entre las pymes de sus zonas.

\begin{abstract}
"La otra [opción] es que la empresa nos pague a nosotros. Que nos paguen a nosotros el costo de ese curso, y ellos se lo deduzcan, nosotros tampoco tenemos problemas. Pero nosotros tenemos una limitación ahí, entre comillas, a la hora de facturar. Por esa pega, porque si yo tuviera aquí la artillería del centro privado X, ¿me explico? Llama a las empresas: ‘¿Qué cursos...? Sí, tenga. Pues yo te facturo, tal, y tú te lo descuentas de tal'." (FP4, p 9)

"Claro, nosotros, toda esa colaboración tenemos, pero lo bonito sería que fuese el centro el que facturara, y que luego, bueno, pues cobrase el profesor, o lo que fuese, pero que saliera del centro, que figurara Educación. Y al final, simplemente es una relación a título personal, entre el profesor y la empresa. Aunque se realiza todo a través del centro... Al final, pues las personas no pueden facturar, pero siendo profesor, pues hasta unos 3000 euros, o así, cantidades pequeñas, puedes llegar a facturar con el DNI. Pero lo bueno sería que entrase Educación ahí. Porque estás dando un curso, y tienes relación, o una colaboración con una empresa... Se podría llegar a un cauce, o alguna vía... (FP8, pp 8-9)

"Hemos empezado ahora. La asociación empresarial comarcal X ha empezado [a fomentar] ese modelo de formación, y se lo está enseñando a las empresas, y las empresas lo están utilizando. Desde septiembre hasta mitad, el primer curso... Lo que nos parece, bueno, no nos parece mal, pero nosotros estamos limitados a ese número de horas... Y luego tampoco podemos facturar" (FP8, p 28)
\end{abstract}

En suma, cabe afirmar que el potencial de la formación continua, como forma avanzada de relación entre centros de FP y empresas, no está siendo suficientemente aprovechado y desarrollado en Navarra debido a los problemas de falta de coordinación entre sistema de FP y el sistema de Formación para el Empleo, así como a las rigideces internas de aquél. No obstante, allá donde existen, las relaciones entre los centros de FP y las pymes industriales basadas en la formación continua son bien valoradas por las empresas. De estas relaciones se derivan múltiples beneficios para los centros de FP, como son, por un lado, un mejor conocimiento de las necesidades de las empresas y, por otro, los recursos materiales que se derivan de la formación para trabajadores subvencionada, que son empleados para la renovación y adquisición de equipos.

\title{
7. FP E INNOVACIÓN
}

Además de las principales misiones de los centros de FP (la cualificación de los estudiantes y la recualificación de los trabajadores), una tercera misión, "no tradicional", de 
los centros sería el apoyo (más explícito) a la innovación en las pymes. El Decreto Foral 54/2008, en su artículo 26, señala el carácter estratégico de la innovación tecnológica (además de la puramente didáctica), e indica que se constituirá un sistema de vigilancia tecnológica y se fomentarán los proyectos de innovación ${ }^{10}$.

A la luz de los resultados obtenidos en la encuesta realizada, se desprende que el papel de los centros de FP en proyectos formales de innovación con las empresas es realmente reducido en este momento en Navarra. Un 5\% de las empresas tienen algún centro de FP como colaborador en programas de mejora de gestión/organización y tan sólo un 1,7\% ha realizado con este tipo de centros algún proyecto (formal) de innovación técnica.

Tabla 6.

\section{Contribución de la FP con servicios para la innovación (N=59)}

\begin{tabular}{lcc} 
& Relación (\%) & $\begin{array}{c}\text { Satisfacción } \\
(0-10) \text { (Media) }\end{array}$ \\
\hline $\begin{array}{l}\text { Colaboran con nosotros como asesores/consultores en } \\
\text { programas de mejora de la gestión / mejoras organizativas }\end{array}$ & 5,1 & 7,00 \\
$\begin{array}{l}\text { Colaboran con nosotros en proyectos (formales) de } \\
\text { innovación técnica }\end{array}$ & 1,7 & 5,00 \\
$\begin{array}{l}\text { Utilizamos sus instalaciones /maquinaria para efectuar } \\
\text { pruebas, ensayos, etc. }\end{array}$ & 10,2 & 7,67 \\
\hline
\end{tabular}

Los contactos entre centros de FP y pymes que pueden redundar en mejoras e innovaciones en éstas pueden tomar distintas formas. Así, cabe señalar que un $10 \%$ de las empresas hayan utilizado las infraestructuras técnicas de los centros de FP para pruebas o ensayos y manifiesten una alta satisfacción $(7,7)$ con este servicio.

Las entrevistas nos han permitido corroborar la extensión, a día de hoy limitada, de los proyectos formales de innovación, apoyados por una línea incipiente de la Administración regional. Sin lugar a dudas, la crisis ha frenado algunas de estas posibles iniciativas entre las empresas. No obstante, se dan casos exitosos que pueden servir de modelo de la potencialidad de este tipo de colaboración.

"En los centros de FP, hay un material con el que nadie cuenta, y es el material humano de los profesores. Hay personas muy, muy válidas, con muchas ganas de hacer algo, que podrían participar en proyectos..." (E7, p.14)

"Sí que podríamos colaborar, y no lo hacemos. Culpa de ambos." (E10, p.9)

"¿Servicios a la empresa? Hay pequeñas pruebas, que suelen hacer con empresas que no tienen recursos técnicos como para hacer algunos ensayos. Alguna cosilla sí que se ha hecho. Pero, más allá, el planteamiento, sería entrar... Claro, las empresas, ya sabes, lo que quieren es hacer una cosa rápida. Y a nosotros más nos interesaría entrar en temas de colaboración en proyectos de innovación" (FP10, p 25)

\footnotetext{
${ }^{10}$ En el momento de la realización del trabajo de campo de este estudio se habían realizado cuatro convocatorias anuales de proyectos de innovación.
} 


\begin{abstract}
"Contactamos con una empresa que se dedicaba a hacer algo de visión. Entonces vino uno, el representante, un poco, el técnico, y yo le dije: 'bueno, a ver, algún problema que tengáis en vista que no se pueda resolver'. Y entonces dijo: 'pues tengo este tema de las cartas'. Y así empezó el tema. Presentamos un proyecto de innovación, y nos lo aceptaron... En la cadena de esta empresa tienen puesto el sistema [que desarrollamos] en dos o tres sitios" (FP12, pp 17 y 22)
\end{abstract}

En la encuesta realizada a las pymes industriales, las empresas han valorado la importancia de los trabajadores con perfil de FP en la innovación mediante una escala 0-10 y en cuatro aspectos concretos, a saber: el desarrollo de nuevos productos, el desarrollo de nuevos procesos, la implantación de nuevas tecnologías y la implantación de nuevos métodos organizativos.

Tabla 7.

Valoración de la importancia de los trabajadores de FP en los procesos de innovación FP (\%) ( $\mathbf{N}=81$ empresas)

\begin{tabular}{lcccc}
\hline & $\begin{array}{c}\text { Desarrollo de } \\
\text { nuevos productos }\end{array}$ & $\begin{array}{c}\text { Desarrollo de } \\
\text { nuevos procesos }\end{array}$ & $\begin{array}{c}\text { Implantación } \\
\text { de nuevas } \\
\text { tecnologías }\end{array}$ & $\begin{array}{c}\text { Implantación } \\
\text { nuevos métodos } \\
\text { organizativos }\end{array}$ \\
\hline $0-2$ & 27,2 & 13,6 & 21,0 & 25,9 \\
$3-4$ & 14,8 & 13,6 & 11,1 & 11,1 \\
$5-6$ & 18,5 & 29,6 & 24,7 & 27,2 \\
$7-8$ & 32,1 & 30,9 & 32,1 & 27,2 \\
$9-10$ & 4,9 & 8,6 & 7,4 & 4,9 \\
NS/NC & 2,5 & 3,7 & 3,7 & 3,7 \\
\hline Total & 100,0 & 100,0 & 100,0 & 100,0 \\
\hline Media valoración & $\mathbf{4 , 6 2}$ & $\mathbf{5 , 4 9}$ & $\mathbf{5 , 1 4}$ & $\mathbf{4 , 7 1}$ \\
\hline
\end{tabular}

Para todos los aspectos, las valoraciones positivas de importancia (5-10) superan a las negativas, es decir, para la mayoría de los responsables de las pymes industriales navarras los trabajadores con perfil de FP desempeñan un papel activo en los procesos de innovación. El tipo de innovación para el que comparativamente se concede mayor importancia a la participación de los trabajadores es el desarrollo de nuevos procesos (media de 5,49) y la implantación de nuevas tecnologías (media de 5,14), y el aspecto en el que tienen menor consideración los trabajadores de FP es el relativo al desarrollo de nuevos productos $(4,62$ de importancia media). Es de destacar que no menos de un $32 \%$ (y hasta un $40 \%$ ) de las empresas encuestadas afirman que los trabajadores con perfil de FP tienen una considerable importancia para alguno de los aspectos de la innovación tratados en este trabajo (valoraciones 7-10). No obstante, a la vista de estos datos, cabe afirmar que el reconocimiento de la aportación de los trabajadores técnicos a la innovación en este momento es más bien incipiente o bajo. 
La evidencia cualitativa, de tipo exploratorio, recogida en las empresas apunta ejemplos interesantes de participación de los trabajadores de FP en tareas relacionadas con la innovación, fundamentalmente en lo relativo a montajes, puestas en marcha, mantenimientos, desarrollos de nuevos procesos y demás actuaciones que suponen la implementación de aquello que viene definido de las oficinas técnicas o ingenierías de proceso. El estudio de estas formas de participación debería ser objeto de futuras investigaciones.

"Son gente que tienen una cualificación. Es decir, ellos saben que tienen que hacer esta máquina, pero tienen su propio pensamiento, su propio razonamiento, y por supuesto, ellos piensan cómo mejorar, o cómo realizar... Eso lo tienen que realizar de la mejor manera posible. O sea, no se les da todo hecho, sino que ellos aportan en cada proyecto." (E2, p.9)

"En este grupo de desarrollo de nuevas ideas para determinados clientes importantes hay un montador. ¿Qué pasa?, que son máquinas que quien mejor las conoce es el que las ha montado, y es el que te va a aportar la idea, y dice "oye, pero este tema, lo podríamos hacer de esta otra manera, ¿no?’.(E2, p12)

"Y aquí, por ejemplo, intentamos que el nuevo personal sea personal de Formación Profesional. Es gente hábil y cuando empiezan a tener experiencia están constantemente: "Oye, vamos a cambiar el proceso...". No te estoy diciendo que los empleados estén pensando en dar vuelta a toda la empresa, ¿no?, pero sí que en su puesto de trabajo cambian mucho, cambian mucho y piensan mucho." (E12, p.8)

"En la definición del producto, no demasiado. En los procesos de fabricación, seguro que tienen algo que decir, ¿no?, en cómo se hacen las acciones de ensamblaje... En la parte que nos toca en Ingeniería, donde se define el producto, pues no excesivamente." (E15, p.10)

"Hay una retroalimentación, sobre todo, de las puestas en marcha, porque muchas veces se enfrentan a problemas que no considerábamos ninguno de nosotros. Y todo eso se retroalimenta. También aportan lo suyo." (E7, p3)

\section{CONCLUSIONES}

En este trabajo hemos mostrado que los centros de FP de la CFN tienen un nivel muy importante de relación con el entorno empresarial regional. El principal nexo a este respecto son las relaciones continuas, en un contexto de cercanía, reciprocidad y confianza, entre los tutores de los centros y los tutores de las empresas. Estas relaciones posibilitan la inserción del capital humano en las empresas y la adaptación del curriculum educativo de los centros a las necesidades empresariales. Los centros de FP de Navarra son, por tanto, una estructura clave en la provisión de mano de obra cualificada en la región, siendo muy bien valorados a este respecto por las empresas. El principal punto de mejora a este respecto sería la extensión, a día de hoy limitada, del programa de estancias de los profesores en las empresas, que posibilite un mayor contacto de los docentes en general (y no sólo de los tutores de prácticas) con la realidad empresarial.

En relación con la formación continua (recualificación de los trabajadores con empleo o desempleados), un elemento central en regiones internacionales de referencia, los centros navarros de FP efectúan una labor considerable y bien valorada por las empresas. En el caso específico de la formación a la carta, se demuestra una manera de relación con 
los centros de FP menos frecuente pero más intensa (ya que la práctica totalidad de las empresas que realizan este tipo de formación tiene ya otros tres o más tipos de relaciones diferentes con los centros) y con unos niveles de satisfacción por parte de las empresas muy altos. Con todo, cabe señalar que existen importantes barreras en el desarrollo de la formación continua en los centros de FP, tanto internas como externas a los mismos. Entre las internas hay que mencionar la falta de autonomía de los centros, las limitaciones en la normativa vigente y la falta de incentivos para los docentes, barreras que son también señaladas por la literatura internacional. Entre las externas está la falta de coordinación entre los departamentos de Educación y Empleo del gobierno regional, así como el bajo nivel de financiación desde el Servicio Navarro de Empleo hacia los centros de FP. El desarrollo del modelo de "centro integrado de FP" que atienda a las diferentes funciones, previsto por la normativa reciente, tanto estatal como autonómica, se haya por tanto ralentizado.

La tercera "misión" de los centros de FP (o segunda misión "no tradicional" de los mismos), relacionada con la prestación de servicios de asistencia técnica a las empresas, se encuentra, a la luz de la evidencia aportada en este trabajo, poco desarrollada en Navarra. Algunas empresas utilizan las instalaciones y maquinaria de los centros para hacer pruebas y ensayos pero, en general, el papel de los centros como facilitadores de la innovación en las empresas es, hoy por hoy, reducido. A este respecto, la convocatoria de proyectos de innovación que ha lanzado en los últimos años el gobierno regional ha tenido hasta el momento un alcance limitado.

Desde el punto de vista de las empresas, se detecta un reconocimiento incipiente pero insuficiente de las aportaciones que los trabajadores técnicos realizan o pueden realizar en los procesos de innovación, aunque la evidencia cualitativa recogida muestra interesantes ejemplos al respecto. Es éste un aspecto que debe ser estudiado en profundidad en investigaciones futuras, tal y como se señala también en el ámbito internacional.

En general, a pesar de que la FP ha jugado un papel importante en el desarrollo económico de Navarra, en la actualidad se encuentra desligada de las infraestructuras de I+D (centros tecnológicos y universitarios) potenciadas en los últimos años por el gobierno regional.

A pesar de la buena valoración de los ciclos de FP superior entre las empresas y del significativo número de alumnos/as que acceden a ingenierías técnicas universitarias tras completar un ciclo superior (estos alumnos serían muy bien valorados por las empresas), la relación entre el sistema de FP y el universitario es, según las entrevistas realizadas, inexistente, no habiendo coordinación entre estas dos instituciones, que constituyen dos mundos diferentes. La inclusión de ambas parcelas bajo una única dirección general en el Gobierno de Navarra (Formación Profesional y Universidades) no parece haber generado sinergias transversales ${ }^{11}$. En cuanto a los centros tecnológicos, la relación es, así mismo, inexistente por lo que, al igual que en otros países, quedan sin aprovechar posibles sinergias señaladas por la literatura internacional.

Como conclusión general, cabe señalar que de las relaciones entre empresas y centros de FP se derivan importantes beneficios para empresas y centros formativos y, en última instancia, para el desarrollo regional. En el futuro es necesario un mayor reconocimiento de

\footnotetext{
${ }^{11}$ El problema principal es también de ámbito estatal, y se manifiesta en aspectos como las dificultades en las convalidaciones de asignaturas de alumnos/as que acceden a la universidad desde los grados superiores de FP.
} 
la interrelación entre el sistema de formación y el sistema regional de innovación, un nexo frecuentemente olvidado por las políticas de innovación (y por algunos de sus actores). Es necesario también ampliar el concepto de innovación imperante, excesivamente lineal y jerárquico, y profundizar en el papel que los trabajadores técnicos cualificados pueden tener en los procesos de innovación de las empresas.

Este trabajo exploratorio se ha basado en la visión que tienen los actores (responsables educativos, directivos de empresas y otros agentes sociales) sobre la aportación de los centros de FP a las pymes industriales. Se precisan de investigaciones empíricas más amplias, así como análisis comparativos que tengan en cuenta los contextos institucionales, que profundicen en el estudio de la contribución de la FP a la cualificación de los trabajadores y a la transferencia de conocimiento a las empresas, al objeto de aumentar la comprensión de las relaciones entre cualificaciones técnicas intermedias e innovación.

\section{REFERENCIAS BIBLIOGRÁFICAS}

ASHEIM, B. y GERTLER M (2005): "The Geography of Innovation: Regional Innovation Systems", en FAGEBERG, J., MOWERY, D. y NELSON, R (Eds.), The Oxford Handbook of Innovation, Oxford, Oxford University Press, pp. 291-317

ASHEIM, B.T. y COENEN, L. (2005):"Knowledge bases and regional innovation systems: comparing nordic clusters", Research policy, Vol. 34, N 8, pp.173-1190

BASTERRETXEA, I.; GONZÁLEZ, A.; SAIZ, M.; y SIMÓN, L. (2002): Colaboración entre los centros de Formación Profesional y las empresas en la Comunidad Autónoma Vasca. Leioa, Servicio Editorial de la UPV/EHU.

BOSCH, G. y CHAREST, J. (2008): "Vocational training and the labour market in liberal and coordinated economies", Industrial Relations Journal, Vol. 39, No 5, pp 428-447.

BRACZYK, H.; COOKE, P. y HEIDENREICH, R. (EDS.) (1996): Regional Innovation Systems. The Role of Governances in a Globalized World, Londres, University College Londres Press.

BRINTON, M.C. (2005): "Education and the Economy", en SMELSER, N.J. y SWEDBERG, R., Hanbook of Economic Sociology, New York, Russel Sage Foundation, pp. 575-602

BROWN, P.; GREEN, A. y LAUDER, H. (2001): High Skills: Globalisation, Competitiveness and Skill Formation. Oxford. Oxford University Press.

CEDEFOP (2010): "La modernización de la formación profesional". Oficina de Publicaciones de la Unión Europea. Luxemburgo. http://www.cedefop.europa.eu/EN/Files/9013_es.pdf (disponible en febrero de 2011)

CONSEJO ESCOLAR DE NAVARRA Y DEPARTAMENTO DE EDUCACIÓN DEL GOBIERNO DE NAVARRA (2010): Informe del Sistema Educativo de Navarra 2009-2010, Pamplona, Gobierno de Navarra.

COOKE, P.; GOMEZ, M. y ETXEBARRIA, G. (1997): "Regional Innovation Systems: Institutional and Organizational Dimensions", Research Policy, Vol 26, No s 4-5, pp. 475-491.

COOKE, P. y MORGAN. K. (1998): The associational economy: Firms, regions and innovation, Oxford, Oxford University Press.

CURTAIN, R. (2004): "Innovation and Vocational Education and Training”, en Dawe, S. (ed), Vocational Education and Training and Innovation: Research Readings, Adelaida, SA, Australia, National Centre for Vocational Education Research (NCVER), pp. 42-58.

DECRETO FORAL 54/2008, de 26 de mayo, por el que se regula la ordenación y el desarrollo de la formación profesional en el sistema educativo de la Comunidad Foral de Navarra (Boletín Oficial de Navarra de 20 de junio de 2008) 
DOLOREUX, D. (2003): "Regional innovation systems in the periphery: the case of Beauce in Quebec (Canada)", International Journal of innovation management, Vol. 7, $\mathrm{N}^{\circ}$ 1, pp. 67-94

EDQUIST, C. (1997): Systems of Innovation: Technologies, Institutions, and Organizations, Londres, Pinter.

ESTEVEZ-ABE, M.; IVERSEN, T. y SOSKICE, D.W. (2001): "Social protection and the formation of skills: a reinterpretation of the welfare state", en HALL, P.A., SOSKICE, D.W. (Eds.), Varieties of Capitalism: The institutional Foundations of Comparative Advantage, Oxford University Press, pp. 145-183

GEBAUER, A.; WOON NAM, C. y PARSCHE, R. (2005): "Regional technology policy and factors shaping local innovation networks in small German cities", European Planning Studies, Vol. 13, $\mathrm{N}^{\circ} 5$, pp. 661-683

GOBIERNO DE NAVARRA (2001): Plan Navarro para el desarrollo de la Formación Profesional 2001-2004. Pamplona, Consejo Navarro de la Formación Profesional.

GOBIERNO DE NAVARRA (2005): Acuerdo de 4 de abril de 2005 del Gobierno de Navarra, por el que se establecen las directrices para el desarrollo del Sistema de Cualificaciones y Formación Profesional en Navarra. Pamplona, Gobierno de Navarra.

GROTZ, R. y BRAUN, B. (1997): “Territorial or transnational networking: spatial aspects of technology oriented cooperation within the German mechanical Engineering Industry", Regional Studies, Vol. 31, N 6 , pp. 545-557.

GUTHRIE, H. y DAWE, S. (2004): "Overview”, en DAWE, S. (ed), Vocational Education and Training and Innovation: Research Readings, Adelaida, SA, Australia. NCVER, pp. 10-19

HOMS, O. (2008): La Formación Profesional en España. Hacia la sociedad del conocimiento, Barcelona, Fundación "la Caixa".

ISAKSEN, B.T. y ASHEIM, A. (2003): "SMEs and the regional dimension of innovation", en ASHEIM, B. T.; ISAKSEN, A.; NAUWELAERS C. y TÖDTLING, F. (eds.): Regional innovation policy for small-medium enterprises, Cheltenham, Edwar Elgar, pp. 21-46.

JENSEN, M.B.; JOHNSO, B.; LORENZ, E. y LUNDVALL, B.A. (2007): "Forms of knowledge and modes of innovation", Research Policy, Vol. 36, Nº5, pp. 680-693.

KAUFMANN, A. y TODTLING, F. (2003): "Innovation pattern of SMEs", en B. ASHEIM, A. ISAKSEN, C. NAUWELAERS y TÖDTLING, F. (Eds.), Regional innovation policy for smallmedium enterprises, Cheltenham, Edwar Elgar, pp. 78-115.

LAVÍA, C.; OTERO, B.; OLAZARAN, M. y ALBIZU, E., (2011), "Innovación y territorio: una encuesta a pequeñas y medianas empresas (pymes) industriales". Revista Internacional de Sociología, 69 (2), pp. 461-486

LUNDVALL, B.A. (ED.), (1992): National Systems of Innovation: Towards a Theory of Innovation and Interactive Learning, Londres, Pinter.

LUNDVALL, B.A. (2002): Innovation, Growth and Social Cohesion: The Danish Model, Cheltenham, Edward Elgar.

LUNDVALL, B.A. (2009): Innovation and Competence Building in the Learning Economy - Implications for Innovation Policy, Aalborg Univ, Dep of Business Studies, Working Paper Series No. 2

LUNDVALL, B.A. y CHRISTENSEN J.L. (2003): "Broadening the Analysis of Innovation Systems - Competition, Organisational Change and Employment Dynamics in the Danish System", en CONCEICAO, P., HEITOR, M.V. y LUNDVALL, B.A., Innovation, Competence Building and Social Cohesion in Europe. Towards a Learning Society, Cheltenham, Edward Elgar, pp 144179.

LUNDVAL, B. A. y LORENZ, E. (2007): "Modes of Innovation and Knowledge Taxonomies in the Learning economy", CAS workshop on Innovation in Firms, Oslo.

LUNDVALL, B.A.; RASMUSSEN, P. y LORENZ, E. (2008): "Education in the Learning Economy: A European Perspective”, Policy Futures in Education, Vol 6, № 6, pp 681-700. 
MASKELL, P. (2001), "Social capital, innovation and competitiveness", en S. Baron, J. Field, y T. Schuller (Eds.), Social Capital: Critical perspectives, Oxford, Oxford University Press, pp.111123.

MASKELL, P. y MALMBERG, A. (1999): "Localised learning and industrial competitiveness.", Cambridge Journal of Economics, Vol 23, No 2, 167-186.

MOODIE, G. (2006): "Vocational education institutions' role in national innovation", Research in Post-compulsory Education, Vol 11, No 2, pp 131-140.

NELSON, R.R. (ED.) (1993): National Innovation Systems: A Comparative Analysis, Oxford, Oxford University Press.

OECD (2001): The Well-being of Nations, The Role of Human and Social Capital, Paris. OECD.

OECD (2009): Education at a Glance, Paris: OECD.

OLAZARAN, M.; ALBIZU, E. y OTERO, B. (2011): "Cooperación y creación de conocimiento: innovación en las pequeñas y medianas empresas industriales", en GONZÁLEZ DE LA FE, T. y LÓPEZ PELÁEZ, A., Innovación, conocimiento científico y cambio social. Ensayos de sociología ibérica de la ciencia y la tecnología, Madrid, Centro de Investigaciones Sociológicas, pp. 131-162.

ROSENBERG, N. (1994): Exploring the Black Box: Technology and Economics, Cambridge, Cambridge University Press.

ROSENFELD, S. (1998): Technical colleges, technology deployment and regional development, Modena, OECD.

ROSENFELD, S. (1998b): Community college/cluster connections: Specialization and competitiveness in the US and Europe, Nueva York: Community College Research Centre, Columbia University.

RUBIO, A y ARAGÓN, A. (2002): "Factores explicativos del éxito competitivo. Un estudio empírico en la pyme", Cuadernos de Gestión, Vol. 2, Nº1, pp. 49-63.

STORPER, M. (1997): The regional economy, Nueva York, Guilford Press.

TETHER, B.; MINA, A.; CONSOLI, D. y GAGLIARDI, D. (2005): A Literature Review on Skills and Innovation. How Does Successful Innovation Impact on the Demand of Skills and How Do Skills Drive Innovation?, Manchester: ESRC Centre for Research on Innovation and Competition (CRIC), Universidad de Manchester.

TONER, P. (2009): Workforce Skills and Innovation: An Overview of Major Themes in the Literature, Paris, OCDE.

TONER, P.; MARCEAU, J.; HALL, R. y CONSIDINE, G. (2004): "Innovation agents: Vocational education and training skills in the present and future Australian innovation system", en DAWE, S. (ed), Vocational Education and Training and Innovation: Research Readings, Adelaida, Australia. NCVER, pp 84-105.

TONER, P.; TURPIN, T.; WOOLLEY, R. y LLOYD, C. (2010) The Role and Contribution of Tradespeople and Technicians in Australian Research \& Development: An Exploratory Study, University of Western Sydney: Centre for Innovation Studies, 2010.

VELLUZI, N.D. (2010): "Community colleges, clusters and competition: A case from Washington Wine Country”, Regional Studies, Vol. 44, №2, pp. 201-214.

VON HIPPEL, E. (1988): The Sources of Innovation, Nueva York, Oxford University Press.

WHITTINGHAM, K.; FERRIER, F. y TROOD, C. (2004): "Vocational education and training and the commercialisation of Australian research", en DAWE S (ed.), Vocational Education and Training and Innovation: Research Readings, Adelaida, Australia. NCVER, pp 106-117. 
